Tema: Engenharia de superfície

\title{
INFLUÊNCIA DO TRATAMENTO SUPERFICIAL DE SHTPN NO COMPORTAMENTO TRIBOLÓGICO DO AÇO INOXIDÁVEL FERRÍTICO AISI 409*
}

\section{Resumo}

\author{
Rafael Amboss Pinto \\ Vanessa Hungria Amboss Pinto ${ }^{2}$ \\ Paulo César Borges 3 \\ Giuseppe Pintaúde ${ }^{4}$
}

O objetivo do SHTPN (Solution Heat Treatment after Plasma Nitriding) é a adição de nitrogênio em solução sólida na superfície, formando austenita durante o tratamento térmico de solubilização, possibilitando a obtenção de martensita de nitrogênio após o resfriamento. Foram ensaiadas amostras nas condições: de fornecimento (microestrutura ferrítica); nitretada (com camada de compostos); após SHTPN (martensita não revenida); e SHTPN seguido de quatro temperaturas de revenimento (martensita revenida). Os ensaios tribológicos foram realizados em placa contra esfera de metal duro, movimento recíproco. Os mecanismos de desgaste foram caracterizados por microscopia eletrônica de varredura (MEV) e as profundidades das pistas foram medidas em rugosímetro 3D. A condição de maior resistência ao desgaste foi a nitretada. Entretanto, a superfície desgastada destas amostras apresentou microtrincas. O comportamento tribológico da amostra revenida a $600^{\circ} \mathrm{C}$ foi similar ao observado para amostra no estado de fornecimento, pois apresentaram predominância do mecanismo de adesão, com grandes deformações plásticas na superfície. Todas as outras condições apresentaram menores profundidades de desgaste do que 0 estado de fornecimento, com mecanismos predominantes transitando de abrasão para adesão com o aumento da temperatura de revenimento. Na amostra após SHTPN, a baixa capacidade de deformação plástica da camada martensítica não revenida causou fratura do material nas laterais da pista, o que não ocorreu para as condições revenidas.

Palavras-chave: Engenharia de superfície; aço inoxidável ferrítico; martensita de nitrogênio; SHTPN; desgaste por deslizamento.

\section{INFLUENCE OF SHTPN SURFACE TREATMENT ON THE TRIBOLOGICAL BEHAVIOR OF STAINLESS STEEL AISI 409}

\section{Abstract}

The goal of SHTPN (Solution Heat Treatment after Plasma Nitriding) is adding nitrogen in solid solution on the steel surface, leading to austenite formation during the solution heat treatment, allowing formation of nitrogen martensite during cooling. Samples on the following conditions were tested: supply condition (ferritic); nitrided (with compounds layer); after SHTPN (nontempered martensite); and SHTPN followed by four tempering temperatures. The tribological tests were carried out in the plane-sphere configuration, reciprocation movement. The wear mechanisms were assessed by SEM and the wear tracks' depth were measure through $3 \mathrm{D}$ roughness measurement machine. The condition with best wear resistance was the nitrided one. However, the worn surface presented microcracks. The supply sample and the sample tempered at $600^{\circ} \mathrm{C}$ presented the same tribological behavior, showing mainly adhesion, with plastic deformations at the surface. When compared to the supply state, all other conditions shown smaller wear depths, with wear mechanisms from abrasion to adhesion as the tempering temperature increases. The low allowance of plastic deformation by martensite on the SHTPN sample cause fractures on the sides of the wear track, this behavior didn't occur for the tempered conditions.

Keywords: Surface engineering; Ferritic stainless steel; Nitrogen martensite; SHTPN; Sliding wear.

1 Eng. Industrial Mecânico, Eng. de desenvolvimento de produto, Engenharia de desenvolvimento de Produtos Diesel (DS/END1-LA), Robert Bosch Ltda., Curitiba, PR, Brasil.

2 Eng. Industrial Mecânica, Analista de Tecnologia e Inovação, Centro de Informação e Estudos Estratégicos (CIES), Instituto de Tecnologia do Paraná (TECPAR), Curitiba, PR, Brasil.

3 Eng. Mecânico, Dr, Professor, PPGEM, DAMEC, Universidade Tecnológica Federal do Paraná (UTFPR), Curitiba, PR, Brasil.

4 Eng. Metalúrgico, Dr., Professor, PPGEM, DAMEC, UTFPR, Curitiba, Paraná, Brasil.

* Technical contribution to the $1^{\text {st }}$ Workshop on surface treatments of corrosion resistant alloys, July $21^{\text {st }}-25^{\text {th }}$, 2014, São Paulo, SP, Brazil. 


\section{INTRODUÇÃO}

A introdução de nitrogênio em aços inoxidáveis vem sendo utilizada para aumentar o desempenho desses materiais quanto à corrosão. Entretanto, devido à tendência do nitrogênio de formar nitreto com o cromo, quando se aplica nitretação aos aços inoxidáveis, a resistência à corrosão pode ser prejudicada. Pela formação do nitreto de cromo ( $\mathrm{CrN})$ e consequente retirada do cromo da solução sólida, a camada passiva de óxidos pode ser reduzida [1]. Para temperaturas baixas de nitretação isso não ocorre, porém a nitretação deve ser conduzida por um longo tempo [2].

Para se aproveitar das vantagens de uma nitretação com temperatura elevada em aços inoxidáveis (acima de $400^{\circ} \mathrm{C}$ ), porém sem os efeitos indesejados da remoção do cromo de solução sólida, deve-se utilizar um método que previna a formação de CrN ou solubilize-o após a nitretação. O HTGN (High Temperature Gas Nitriding) tem sido estudado como uma alternativa, que consiste em nitretação gasosa a alta temperatura (entre $1000^{\circ} \mathrm{C}$ e $1200^{\circ} \mathrm{C}$ ) para prevenir a formação de nitretos [3]. Outra linha de processamento é o SHTPN, desenvolvido pelo Grupo de Materiais, Tribologia e Superfícies (GrMaTS) da Universidade Tecnológica Federal do Paraná (UTFPR). O processo consiste em duas etapas: Nitretação a Plasma (PN - Plasma Nitriding), com o objetivo de fornecer uma fonte de nitrogênio para o posterior Tratamento Térmico de Solubilização (SHT - Solution Heat Treatment). Para garantir a manutenção do nitrogênio em solução sólida o resfriamento é rápido após a solubilização [4]. Assim, o processo visa aumentar a dureza superficial além de recuperar a resistência à corrosão dos aços inoxidáveis nitretados, devolvendo o cromo, precipitado durante a nitretação, ao estado de solução sólida [1].

$O$ efeito endurecedor do nitrogênio faz com que o SHTPN possa ser uma alternativa para aumentar simultaneamente a resistência à corrosão e ao desgaste. Assumpção [5] concluiu que o SHTPN melhora a resistência à corrosão do aço AISI 409. A literatura apresenta os mecanismos de desgaste predominantes em função da microestrutura de aços inoxidáveis nitretados de forma convencional $[6,7]$. Estudos mostraram que o desempenho de uma camada composta predominantemente por austenita expandida $(\mathrm{YN})$ foi superior às que apresentaram predominantemente nitreto de cromo, em ensaios bloco-sobre-disco [6]. Em ensaios esfera-sobre-disco, Yetim et al. [7] demonstraram que para a austenita expandida o mecanismo principal de desgaste é adesão, enquanto Li et al. [6] observaram abrasão leve e polimento. Há, portanto, uma lacuna a ser investigada no que se refere aos mecanismos predominantes de desgaste, de modo que as microestruturas possam ser projetadas para melhor desempenho.

O objetivo geral desse trabalho é avaliar o comportamento tribológico do aço inoxidável ferrítico AISI 409 processado por SHTPN (após nitretação e solubilização) e com diferentes condições de revenimento posterior (em temperaturas de $300^{\circ} \mathrm{C}$, $400^{\circ} \mathrm{C}, 500^{\circ} \mathrm{C}$ e $600^{\circ} \mathrm{C}$ ). Para tanto, uma comparação é feita com o estado de fornecimento e com a condição de tratamento após nitretação a plasma convencional.

\section{MATERIAIS E MÉTODOS}

A composição química do aço AISI 409, avaliada por espectrometria de absorção atômica, é mostrada na Tabela 1. Sua microestrutura no estado de fornecimento é ferrítica.

\footnotetext{
* Technical contribution to the $1^{\text {st }}$ Workshop on surface treatments of corrosion resistant alloys, July $21^{\text {st }}-25^{\text {th }}$, 2014, São Paulo, SP, Brazil.
} 
Tabela 1. Composição química do aço AISI 409

\begin{tabular}{ccccccccc}
\hline \multicolumn{10}{c}{ Elementos (\% wt) } \\
\hline $\mathbf{C}$ & $\mathbf{N i}$ & $\mathbf{C r}$ & $\mathbf{A l}$ & $\mathbf{M n}$ & $\mathbf{S i}$ & $\mathbf{P}$ & $\mathbf{C u}$ & $\mathbf{V}$ \\
\hline 0,001 & 0,30 & 10,26 & 0,005 & 0,07 & 0,597 & 0,036 & 0,064 & 0,031 \\
\hline
\end{tabular}

Esse material foi escolhido devido aos valores de cromo equivalente ( $\left.\mathrm{Cr}_{\text {eq }}\right)$ e níquel equivalente (Nieq) calculados para a composição química do estado de fornecimento, mostrados nas Equações 1 e 2 [8].

$$
\begin{array}{r}
\text { Creq }=(\% \mathrm{Cr})+(\% \mathrm{Mo})+1,5(\% \mathrm{Si})+0,5(\% \mathrm{Nb})=11,23 \\
\text { Nieq }=(\% \mathrm{Ni})+0,5(\% \mathrm{Mn})+30(\% \mathrm{C})+30(\% \mathrm{~N})=0,37
\end{array}
$$

O diagrama de Schaeffler (Figura 1) foi utilizado como uma aproximação para a escolha do aço a ser estudado: o nitrogênio aumenta o $\mathrm{Ni}_{\text {eq }}$ e permite a formação de austenita no aço ferrítico. Com resfriamento rápido o suficiente, martensita de nitrogênio pode ser formada. Esse efeito foi comprovado em estudos recentes do GrMaTS [9]. As amostras com dimensões de $15 \mathrm{~mm} \times 20 \mathrm{~mm}$ foram obtidas a partir de uma chapa laminada a frio de aproximadamente $2,5 \mathrm{~mm}$ de espessura.

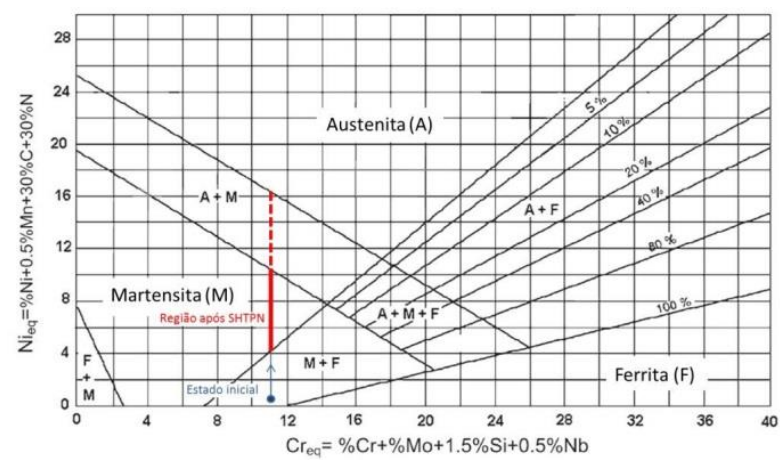

Figura 1. Diagrama de Schaeffler mostrando o ponto de estado de fornecimento do aço AISI 409 e a possibilidade de obtenção de martensita após o SHTPN. Fonte: adaptado de [10].

As amostras foram posicionadas no reator de plasma sobre uma base de aço AISI 304 lixada até grana 600. A nitretação foi feita nas condições mostradas na Tabela 2, que inclui as condições de limpeza por sputtering anterior à nitretação (amostras chamadas $\mathrm{N}$ ).

Tabela 2. Parâmetros de sputtering e nitretação a plasma

\begin{tabular}{c|cc}
\hline Parâmetro & Sputtering & Nitretação a plasma \\
\hline Temperatura $\mathrm{T}\left({ }^{\circ} \mathrm{C}\right)$ & $160 \pm 15$ & $510 \pm 10$ \\
$\mathrm{~T}_{\text {on }}(\mu \mathrm{s})$ & $300 \mathrm{a} 800$ & $50 \mathrm{a} 200$ \\
$\mathrm{~T}_{\text {off }}(\mu \mathrm{s})$ & $50 \mathrm{a} 650$ & $700 \mathrm{a} 900$ \\
Tensão $(\mathrm{V})$ & 400 & 600 \\
Pressão (Torr) & $1,5 \pm 0,2$ & $3,0 \pm 0,2$ \\
Tempo (h) & 1 & 2 \\
Atmosfera gasosa & $\mathrm{H}_{2}$ & $80 \% \mathrm{~N}_{2}+20 \% \mathrm{H}_{2}$ \\
\hline
\end{tabular}

O tratamento térmico de solubilização foi de $1 \mathrm{~h}$ à temperatura de $1200^{\circ} \mathrm{C}$, com posterior resfriamento ao ar (amostras chamadas NS). Após a solubilização, foram realizados revenimentos a $300^{\circ} \mathrm{C}, 400^{\circ} \mathrm{C}, 500^{\circ} \mathrm{C}$ e $600^{\circ} \mathrm{C}$ por $1 \mathrm{~h}$ no mesmo forno com atmosfera controlada, também resfriando ao ar. Essas amostras serão chamadas R300, R400, R500 e R600. Para revelar a microestrutura obtida após os tratamentos, as amostras foram preparadas para metalografia e observadas em um

* Technical contribution to the $1^{\text {st }}$ Workshop on surface treatments of corrosion resistant alloys, July $21^{\text {st }}-25^{\text {th }}, 2014$, São Paulo, SP, Brazil. 
microscópio ótico BX51M da marca Olimpus $^{\circledR}$ equipado com câmera CCD CoolSNAP-procf da marca Media Cybernetics ${ }^{\circledR}$ e software analisador de imagens Image-Pro Plus versão 4.0. Os perfis de microdureza foram obtidos pelo microdurômetro HMV-2, da marca Shimadzu ${ }^{\circledR}$, em ensaios de microdureza Vickers com carga $0,05 \mathrm{kgf}$ e tempo de aplicação $10 \mathrm{~s}$. Para análise de dureza superficial, foi utilizado HV2, com profundidade da identação maior ou igual a 20 vezes o valor de Ra em $\mu \mathrm{m}$ [11]. O acabamento das amostras após os tratamentos foi por retificação plana tangencial de passagem. A profundidade de corte de aproximadamente $300 \mu \mathrm{m}$ permitiu a remoção da camada de óxidos superficial expondo a camada martensítica formada pelo SHTPN.

A avaliação do comportamento sob desgaste consiste em um ensaio tribológico com movimento recíproco em um tribômetro da marca CETR $^{\circledR}$, modelo UMT MultiSpecimen Test System, utilizando uma esfera de carboneto de tungstênio de $4 \mathrm{~mm}$ de diâmetro como contra corpo, que se movimenta transversalmente ao sentido de retificação das amostras. Os parâmetros utilizados para o ensaio tribológico foram: carga de $5 \mathrm{~N}$, frequência de $20 \mathrm{~Hz}$, comprimento da pista de desgaste de $5,5 \mathrm{~mm}$ e tempo total de ensaio de 900 segundos. O valor de 400 segundos ficou estabelecido para início do regime permanente em todos os ensaios. Em cada amostra foram feitas 5 pistas de desgaste e o valor do coeficiente de atrito e desvio padrão é a média aritmética entre esses 5 ensaios. $O$ mecanismo de desgaste em cada pista foi avaliado através de MEV, com o equipamento TM3000, do fabricante Hytashi Technologies ${ }^{\circledR}$. A rugosidade das amostras antes e depois do ensaio de desgaste, bem como a avaliação $3 \mathrm{D}$ da superfície foi realizada através do rugosímetro 3D Talysurf CCI Lite Non-contact 3D Profiler da marca Taylor Hobson ${ }^{\circledR}$. A profundidade de cada pista de desgaste é avaliada de acordo com o valor de Pt, obtido a partir de um perfil 2D transversal ao comprimento da pista. A Tabela 3 mostra os parâmetros $\mathrm{Ra}, \mathrm{Rz}$ e Pt, medidos a partir de um perfil 2D transversal à direção de retificação antes da realização dos ensaios tribológicos.

Tabela 3. Parâmetros antes dos ensaios tribológicos para todas as condições analisadas

\begin{tabular}{|c|c|c|c|c|c|c|c|}
\hline & Fornecimento & Nitretada & SHTPN & $\begin{array}{c}\text { SHTPN } \\
\text { Revenido } 300^{\circ} \mathrm{C}\end{array}$ & $\begin{array}{c}\text { SHTPN } \\
\text { Revenido } 400^{\circ} \mathrm{C}\end{array}$ & $\begin{array}{c}\text { SHTPN } \\
\text { Revenido } 500^{\circ} \mathrm{C}\end{array}$ & $\begin{array}{c}\text { SHTPN } \\
\text { Revenido } 600^{\circ} \mathrm{C}\end{array}$ \\
\hline Ra $[\mu \mathrm{m}]$ & 0,28 & 0,55 & 0,28 & 0,26 & 0,25 & 0,30 & 0,31 \\
\hline $\operatorname{Rz}[\mu \mathrm{m}]$ & 1,89 & 3,52 & 1,85 & 1,82 & 2,04 & 2,16 & 2,01 \\
\hline $\mathrm{Pt}[\mu \mathrm{m}]$ & 2,71 & 4,95 & 2,69 & 2,69 & 2,44 & 3,31 & 2,42 \\
\hline
\end{tabular}

\section{RESULTADOS E DISCUSSÃO}

\subsection{Tratamento Térmico de Solubilização após Nitretação a Plasma (SHTPN) e Revenimentos}

A condição de nitretação na temperatura de $510^{\circ} \mathrm{C}$ e tempo de $2 \mathrm{~h}$, acompanhada de atmosfera rica em nitrogênio, favorece a formação de camada branca em detrimento da camada de difusão. O objetivo é obter uma camada rica em nitretos para servir de fonte de nitrogênio durante a solubilização. Borges e Rocha [1] utilizaram nitretação a plasma para introduzir nitrogênio como fonte para a solubilização, etapa final do SHTPN. A Figura 2 (esquerda) mostra a imagem obtida por MEV da camada após nitretação a plasma. Nota-se claramente camada rica em nitretos, e o material base com microestrutura ferrítica. Na região nitretada foi detectado nitrogênio por análise de EDS, o que não ocorreu na região do material base. A Figura 2 (direita) mostra a microestrutura resultante do processo de SHTPN, que se mantém similar após o revenimento. Houve formação completa de martensita e crescimento de

* Technical contribution to the $1^{\text {st }}$ Workshop on surface treatments of corrosion resistant alloys, July $21^{\text {st }}-25^{\text {th }}, 2014$, São Paulo, SP, Brazil. 
Tabela 4. Valores médios relacionados ao ensaio de desgaste para amostras em diferentes condições

\begin{tabular}{|l|c|c|c|c|c|}
\hline & $\begin{array}{c}\text { Coeficiente de } \\
\text { atrito (COF) }\end{array}$ & $\begin{array}{c}\text { Profundidade } \\
\text { de desgate Pt } \\
{[\boldsymbol{\mu \mathrm { m } ]}}\end{array}$ & $\begin{array}{c}\text { Volume } \\
\text { desgastado } \\
{\left[\mathrm{mm}^{3}\right]}\end{array}$ & $\begin{array}{c}\text { Perda de material } \\
\text { por distância de } \\
\text { deslizamento (Q) } \\
{\left[\mathbf{m m}^{3} / \mathbf{m m}\right]}\end{array}$ & $\begin{array}{c}\text { Dureza de } \\
\text { topo [HV2] }\end{array}$ \\
\hline Fornecimento & $0,53 \pm 0,04$ & $21 \pm 1$ & 0,045 & $4,5 \mathrm{E}-07$ & $150 \pm 2$ \\
\hline Nitretada & $0,55 \pm 0,01$ & $3,4 \pm 0,2$ & 0,003 & $2,9 \mathrm{E}-08$ & $1111 \pm 5$ \\
\hline SHTPN & $0,60 \pm 0,02$ & $13 \pm 2$ & 0,022 & $2,2 \mathrm{E}-07$ & $472 \pm 8$ \\
\hline $\begin{array}{l}\text { SHTPN } \\
\text { Revenida } 300^{\circ} \mathrm{C}\end{array}$ & $0,60 \pm 0,01$ & $13 \pm 1$ & 0,021 & $2,1 \mathrm{E}-07$ & $451 \pm 6$ \\
\hline $\begin{array}{l}\text { SHTPN } \\
\text { Revenida } 400^{\circ} \mathrm{C}\end{array}$ & $0,60 \pm 0,01$ & $12 \pm 1$ & 0,020 & $2,0 \mathrm{E}-07$ & $473 \pm 5$ \\
\hline $\begin{array}{l}\text { SHTPN } \\
\text { Revenida } 500^{\circ} \mathrm{C}\end{array}$ & $0,58 \pm 0,02$ & $13 \pm 2$ & 0,021 & $2,1 \mathrm{E}-07$ & $439 \pm 6$ \\
\hline $\begin{array}{l}\text { SHTPN } \\
\text { Revenida } 600^{\circ} \mathrm{C}\end{array}$ & $0,53 \pm 0,04$ & $24 \pm 1$ & 0,054 & $5,5 \mathrm{E}-07$ & $272 \pm 2$ \\
\hline
\end{tabular}

A pista de desgaste da amostra no estado de fornecimento (amostra $F$ ) é mostrada na Figura 5. A amostra $F$ é a que possui a menor dureza (150HV2) e, consequentemente, apresentou profundidade da pista de desgaste de $21 \pm 1 \mu \mathrm{m}$. Nota-se predominância de desgaste adesivo. A adesão é responsável pelo maior desvio padrão médio do coeficiente de atrito $(0,53 \pm 0,04)$ (Figura 6), gerando flutuações causadas por um processo de constante adesão e desprendimento do material aderido à esfera. Devido à baixa dureza e alta ductilidade da amostra, ocorreu intensa deformação plástica e acúmulo de material nas extremidades da pista.
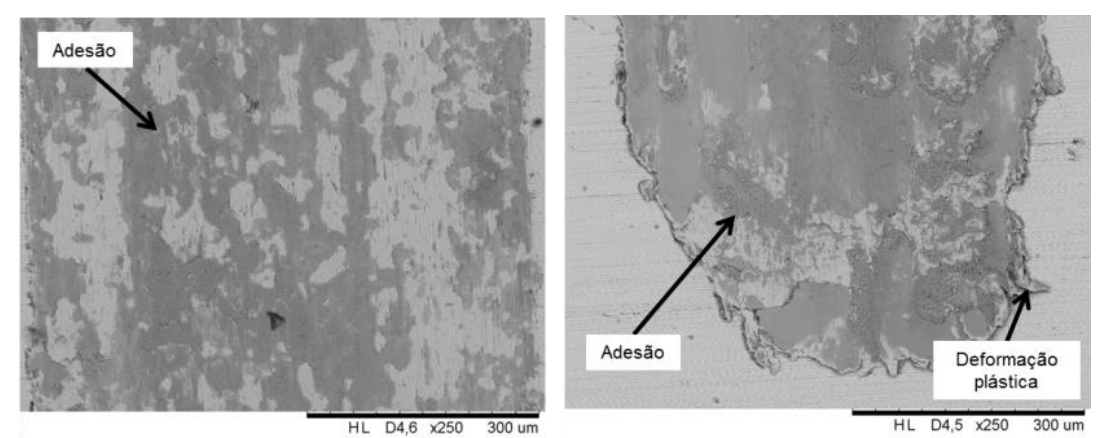

Figura 5. Microscopia eletrônica de varredura do centro (esquerda) e extremidade (direita) da pista de desgaste na amostra do estado de fornecimento. Mecanismo predominante: desgaste adesivo.

A amostra ensaiada logo após a nitretação foi a que sofreu menor desgaste, com profundidade média da pista de apenas $3,4 \pm 0,2 \mu \mathrm{m}$ contra $21 \pm 1 \mu \mathrm{m}$ para a amostra no estado de fornecimento, uma redução de $84 \%$. A condição nitretada não apresentou flutuações ao longo do tempo de ensaio por não ocorrer desgaste adesivo (Figura 6). O mecanismo predominante na amostra $\mathrm{N}$ é o polimento, que consiste em romper as asperezas de forma a homogeneizar a superfície. É possível observar que houve ainda desgaste abrasivo no centro, devido ao desprendimento de partículas duras de nitretos da camada que permanecem na região da pista durante o ensaio tribológico, assim como em [7].

* Technical contribution to the 1st Workshop on surface treatments of corrosion resistant alloys, July $21^{\text {st }}-25^{\text {th }}$, 2014, São Paulo, SP, Brazil. 
A profundidade de material removido no ensaio tribológico para a condição solubilizada foi $38 \%$ menor em relação ao estado de fornecimento. Pelo aspecto geral da pista (Figura 8), o mecanismo predominante foi de desgaste abrasivo. Além desse fenômeno, há também o aparecimento de desgaste por adesão, concentrado no centro da pista e nas extremidades.
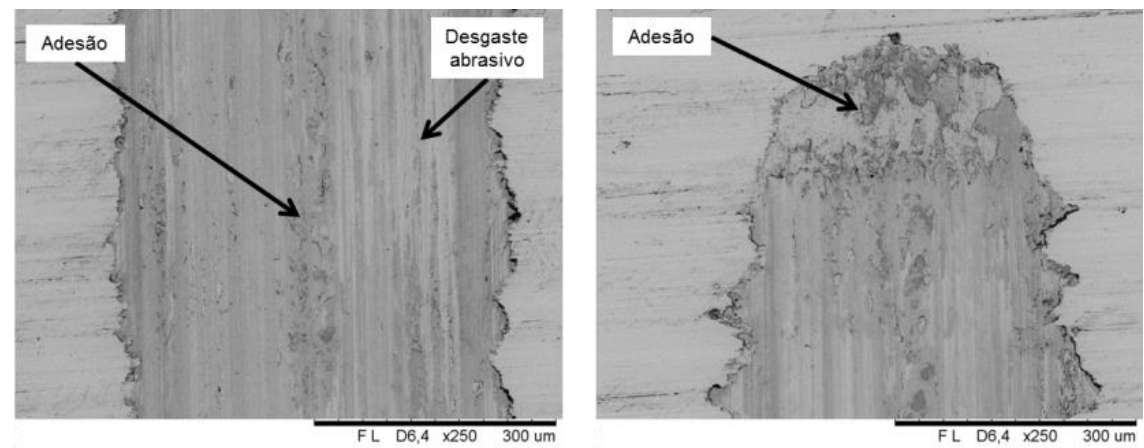

Figura 8. MEV do centro (esquerda) e extremidade (direita) da pista de desgaste na amostra após SHTPN. Mecanismo predominante: desgaste abrasivo.

As amostras revenidas a $300^{\circ} \mathrm{C}, 400^{\circ} \mathrm{C}$ e $500^{\circ} \mathrm{C}$ não apresentaram diferenças consideráveis entre si nos parâmetros de coeficiente de atrito e profundidade da pista, sendo também muito próximos aos obtidos na amostra NS, apresentando uma redução em torno de $40 \%$ de profundidade de material desgastado em relação ao estado de fornecimento. Observou-se, entretanto, que os mecanismos de desgaste diferiram entre as temperaturas de revenimento e em relação à amostra NS. Na amostra R300 predomina abrasão, as regiões com adesão são mais raras que na NS (Figura 9), apesar da dureza sensivelmente mais baixa.
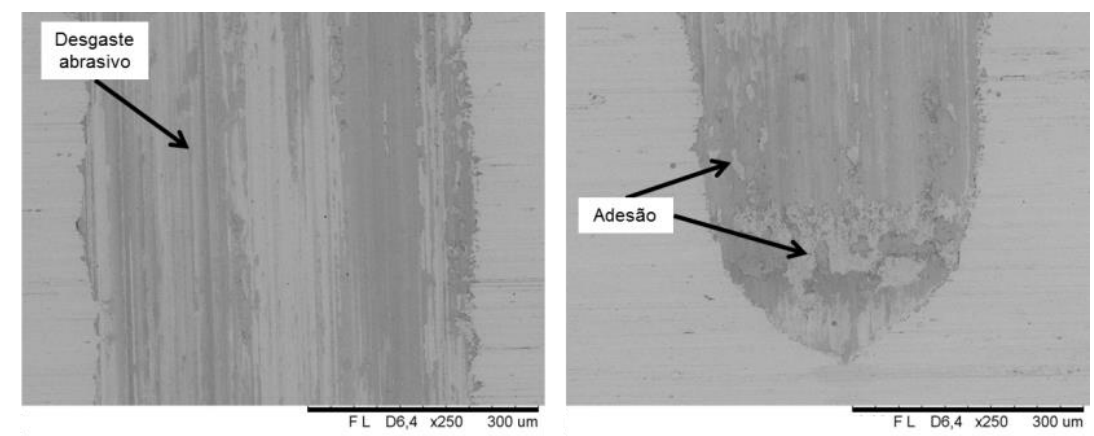

Figura 9. Microscopia eletrônica de varredura do centro (esquerda) e extremidade (direita) da pista de desgaste na amostra após revenimento a $300^{\circ} \mathrm{C}$. Mecanismo predominante: desgaste abrasivo.

Na amostra R400 observou-se que o mecanismo de adesão se tornou mais notável que na R300, com intensidade semelhante à amostra NS. Ainda assim, o mecanismo predominante foi o desgaste abrasivo. O mecanismo de desgaste predominante na amostra R500 foi de adesão, com algumas regiões marcadas por abrasão. Apesar da dureza próxima às condições de R300, R400 e NS, a amostra R500 mostrou características de desgaste intermediárias entre as amostras NS e F (Figura 10). Devido à menor dureza obtida após revenimento a $600^{\circ} \mathrm{C}$, o desgaste observado para essa condição de revenimento foi mais severo e provocou $24 \pm 1 \mu \mathrm{m}$ de profundidade na pista desgastada. Esse valor é muito próximo ao observado para o material no estado de fornecimento $(21 \pm 1 \mu \mathrm{m})$, no qual a dureza de topo foi avaliada em 150 HV2.

* Technical contribution to the $1^{\text {st }}$ Workshop on surface treatments of corrosion resistant alloys, July $21^{\text {st }}-25^{\text {th }}$, 2014, São Paulo, SP, Brazil. 

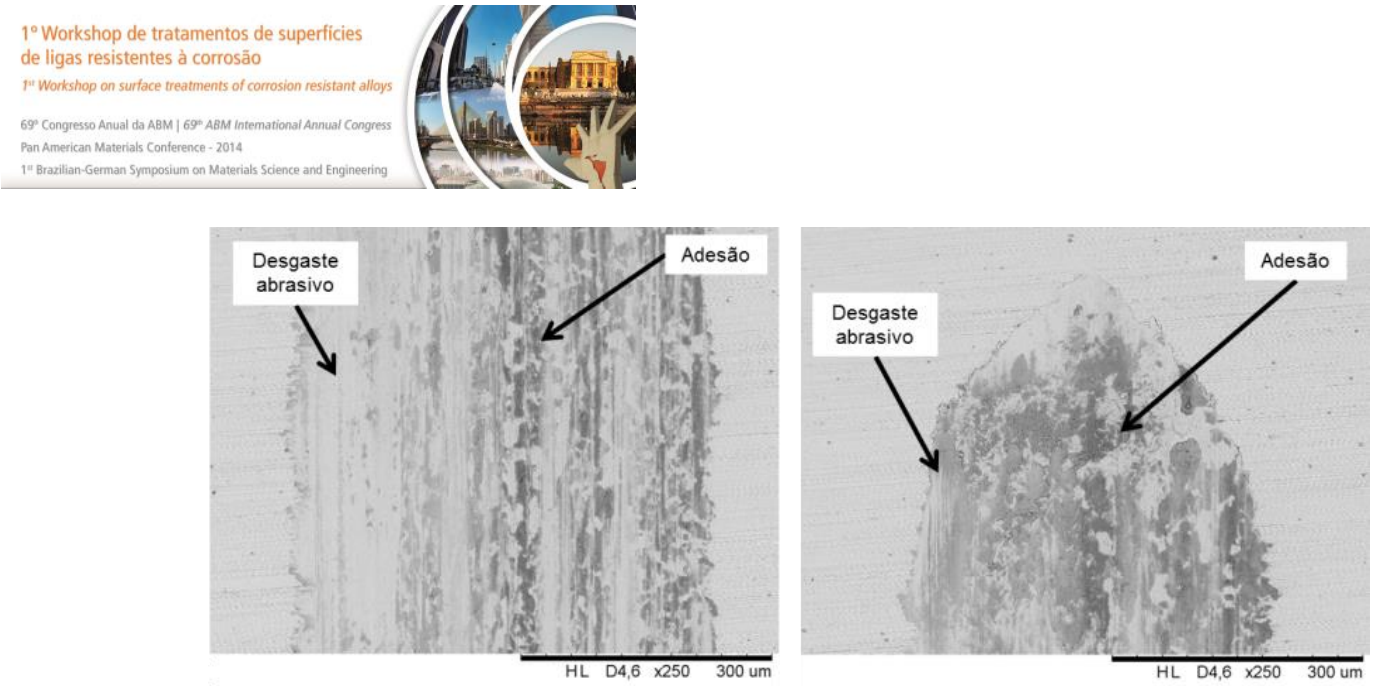

Figura 10. Microscopia eletrônica de varredura do centro (esquerda) e extremidade (direita) da pista de desgaste na amostra após revenimento a $500^{\circ} \mathrm{C}$. Mecanismo predominante: desgaste adesivo.

Os valores de coeficiente de atrito para as amostras R600 e $\mathrm{F}$ também foram iguais e houve significativa similaridade na flutuação desse parâmetro ao longo do tempo de ensaio. $O$ desvio padrão do coeficiente de atrito foi de 0,04 , também maior que nas outras amostras (Figura 11), indicando que a similaridade com a amostra $F$ ocorre também no mecanismo de desgaste, com grande presença de adesão.

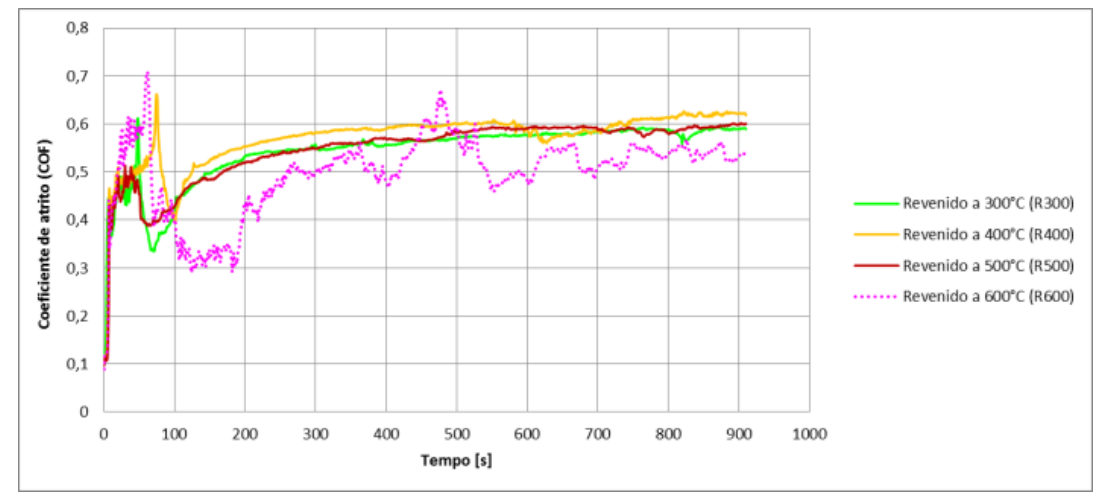

Figura 11. Gráfico de coeficiente de atrito por tempo de ensaio mostrando a maior variação desse parâmetro para a amostra R600 em relação às amostras R300, R400 e R500.

A ocorrência de desgaste adesivo é evidenciada na Figura 11. A deformação plástica nas laterais da pista e o acúmulo de material nas extremidades são um fator de semelhança entre R600 e F. A diferença de dureza das amostras F e R600 não afetou o comportamento sob desgaste. Uma hipótese é que o encruamento devido à carga de ensaio aumentou a dureza na pista em ambas as amostras, e a dureza do metal em contato com a esfera pode ter se tornado similar.
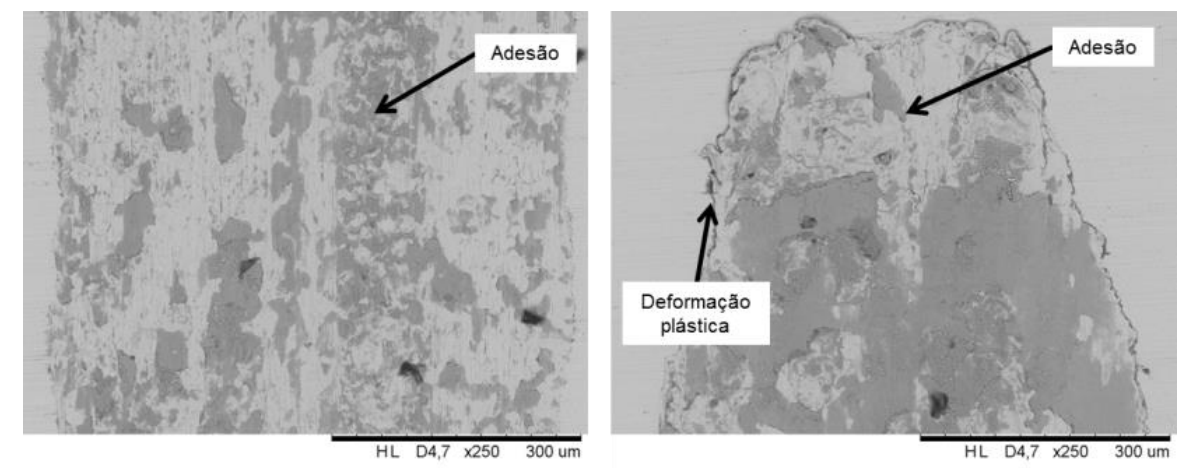

Figura 12. Microscopia eletrônica de varredura do centro (esquerda) e extremidade (direita) da pista de desgaste na amostra após revenimento a $600^{\circ} \mathrm{C}$. Mecanismo predominante: desgaste adesivo.

* Technical contribution to the $1^{\text {st }}$ Workshop on surface treatments of corrosion resistant alloys, July $21^{\text {st }}-25^{\text {th }}$, 2014, São Paulo, SP, Brazil. 


\section{CONCLUSÕES}

- Com o tratamento térmico de SHTPN foi possível a obtenção de uma camada martensítica de nitrogênio de aproximadamente $500 \mu \mathrm{m}$ de profundidade e 450 HV0,05 de microdureza superficial no aço inoxidável ferrítico AISI 409.

- Os revenimentos a temperaturas de $300^{\circ} \mathrm{C}, 400^{\circ} \mathrm{C}$ e $500^{\circ} \mathrm{C}$ resultaram em perfil de microdureza muito similares entre si, com maior dureza de topo no revenimento a $400^{\circ} \mathrm{C}$, possivelmente devido à precipitação de nitretos. Houve uma queda considerável de dureza superficial para a condição a $600^{\circ} \mathrm{C}$, de aproximadamente $450 \mathrm{HV} 2$ para $270 \mathrm{HV} 2$.

- Após o tratamento de SHTPN foi possível observar que as microestruturas das amostras tratadas com SHTPN e tratadas com SHTPN+revenimento nas temperaturas de $300^{\circ} \mathrm{C}, 400^{\circ} \mathrm{C}, 500^{\circ} \mathrm{C}$ e $600^{\circ} \mathrm{C}$ apresentaram estrutura martensítica na camada superficial, ilhas de martensita nos contornos de grão de ferrita na interface com o material base e crescimento de martensita nos contornos de grão da ferrita.

- Após o processo de SHTPN, o aço AISI 409 apresentou melhor desempenho sob desgaste por deslizamento em relação ao estado de fornecimento, com profundidade desgastada aproximadamente $40 \%$ menor. Isso se repete para as condições tratadas com SHTPN+revenimento nas temperaturas de $300^{\circ} \mathrm{C}, 400^{\circ} \mathrm{C}$ e $500^{\circ} \mathrm{C}$. A única condição que não resultou em melhor desempenho sob desgaste foi a revenida a $600^{\circ} \mathrm{C}$, devido à queda significativa de dureza superficial observada para esta temperatura.

- A condição apenas nitretada foi a que resultou em melhor desempenho sob desgaste devido a sua alta dureza superficial em comparação com as outras condições. A profundidade de desgaste foi $84 \%$ menor em relação ao estado de fornecimento. A formação de trincas na amostra nitretada se deve a um alto gradiente de dureza entre camada e substrato. A queda severa de dureza na transição da camada de compostos causa o "efeito casca de ovo". À medida que esse gradiente diminui nas condições solubilizada e revenidas, e com a menor fragilidade da superfície com microestrutura martensítica, o mecanismo de desgaste predominante torna-se adesão, sendo maior para as amostras no estado de fornecimento e revenida a $600^{\circ} \mathrm{C}$.

- A flutuação do coeficiente de atrito ao longo do tempo está relacionada à ocorrência de desgaste adesivo, observado para as amostras de menor dureza: fornecimento e revenida a $600^{\circ} \mathrm{C}$, que têm comportamento sob desgaste semelhante entre si. Essa flutuação do coeficiente de atrito ocorre menos intensamente à medida que se tem menos adesão, não ocorrendo na amostra nitretada, em que ocorreu apenas abrasão.

- De acordo com os mecanismos de desgaste observados, a semelhança nos valores de coeficiente de atrito para as amostras no estado de fornecimento e nitretada é apenas uma coincidência experimental, pois as propriedades mecânicas das amostras são diferentes e os mecanismos de desgaste resultantes não foram os mesmos.

- As amostras revenida a $300^{\circ} \mathrm{C}$ e revenida a $400^{\circ} \mathrm{C}$ são equivalentes quanto ao coeficiente de atrito, profundidade e mecanismo predominante de desgaste: abrasão. Nesse caso a diferença de dureza de topo não teve forte correlação (451 HV2 e 473 HV2 respectivamente).

- A condição revenida a $500^{\circ} \mathrm{C}$ pode ser considerada uma transição entre os comportamentos observados nas amostras revenidas a $300^{\circ} \mathrm{C}$ e a $400^{\circ} \mathrm{C}$

\footnotetext{
* Technical contribution to the $1^{\text {st }}$ Workshop on surface treatments of corrosion resistant alloys, July $21^{\text {st }}-25^{\text {th }}$, 2014, São Paulo, SP, Brazil.
} 
(abrasão) e amostras no estado de fornecimento e revenida a $600^{\circ} \mathrm{C}$ (adesão). Nela os dois mecanismos coexistiram, com leve predominância da adesão.

\section{Agradecimentos}

Agradecemos às empresas Spectroscan, Artiz Matriz e Robert Bosch pelo apoio na parte experimental. Agradecemos ao CNPq pelo financiamento do projeto e à FINEP pelo apoio através do projeto CT-INFRA.

\section{REFERÊNCIAS}

1 Borges PC, Rocha LA. Solution heat treatment of plasma nitrided 15-5PH stainless steel Part I. Improvement of the corrosion resistance. Kovove Mater. 2011; 49:107-117.

2 Larisch B, Brusky U, Spies HJ. Plasma nitriding of stainless steels at low temperatures. Surface and Coatings Technology. 1999; 205-211.

3 Garzón CM, Tschiptschin AP. Nitretação Gasosa em alta Temperatura de Aços Inoxidáveis. Revista Matéria. 2005; 10(4):502-525.

4 Reis RF. Elevação do teor superficial de nitrogênio no aço inoxidável austenítico ISO 5832-1 [tese de doutorado]. Curitiba: Universidade Tecnológica Federal do Paraná; 2007.

5 Assumpção RL. Resistência a corrosão da camada martensítica formada na superfície do aço inoxidável ferrítico 409 tratado por SHTPN [tese de mestrado]. Curitiba: Universidade Tecnológica Federal do Paraná; 2013.

6 Li G, Wang J, Li C, Peng Q, Gao J, Shen B. Microstructure and dry-sliding wear properties of DC plasma nitrided 17-4 PH stainless steel. Nuclear Instruments and Methods in Physics Research. 2008; B(266):1964-1970.

7 Yetim AF, Yildiz F, Alsaran A, Çelik A. Surface modification of 316L stainless steel with plasma Nitriding. Kovove Mater. 2008; 46:105-115.

8 American Society of Materials International (ASM). Welding Brazing and Soldering. Metals Handbook. 1993; 6:1176.

9 Assumpção RL, Borges PC, Martinez VA, Santos L. Martensita de nitrogênio obtida pelo processo de SHTPN em aços inoxidáveis ferríticos. VII Congresso Nacional de Engenharia Mecânica. 2012.

10 Reis RF, Maliska AM, Borges PC. Nitrogen surface enrichment of austenitic stainless steel ISO 5832-1. J Mater Sci. 2011; 46:846-854.

11 International Organization for Standization (ISO). ISO 14577-1:2002, Metallic Materials - Instrumented indentation test for hardness and material parameters - Part 1: Test method.

12 Sung JH, Kong JH, Yoo DK, On HI, Lee DJ, Lee HW. Phase changes of the AISI 430 ferritic stainless steels after high-temperature gas nitriding and tempering heat treatment. Materials Science and Engineering. 2008; A(489):38-43.

13 Jeong $B$, Myung K. Effects of pulse frequency and temperature on the nitride layer and surface characteristics of plasma nitrided stainless steel. Surface and Coatings Technology. 2001; 137: 249-254.

* Technical contribution to the $1^{\text {st }}$ Workshop on surface treatments of corrosion resistant alloys, July $21^{\text {st }}-25^{\text {th }}$, 2014, São Paulo, SP, Brazil. 